\title{
Kernos
}

Revue internationale et pluridisciplinaire de religion grecque antique

19 | 2006

Varia

\section{Jan BREMMER, La religione greca}

\section{Gabriella Pironti}

\section{(2) OpenEdition}

\section{Journals}

\section{Édition électronique}

URL : https://journals.openedition.org/kernos/471

DOI : $10.4000 /$ kernos.471

ISSN : 2034-7871

Éditeur

Centre international d'étude de la religion grecque antique

Édition imprimée

Date de publication : 1 janvier 2006

Pagination : 459-461

ISSN : 0776-3824

Référence électronique

Gabriella Pironti, « Jan Bremmer, La religione greca », Kernos [En ligne], 19 | 2006, mis en ligne le 22 mars 2011, consulté le 24 août 2022. URL : http://journals.openedition.org/kernos/471 ; DOI : https:// doi.org/10.4000/kernos.471 


\section{Comptes rendus et notices bibliographiques}

Jan Bremmer, La religione greca, ouvrage traduit de l'anglais par M. Rosetti, post-face de G. Casadio, Cosenza, Ed. Lionello Giordano, 2002. 1 vol. 14,5 × 20,5 cm, 190 p. (Biblioteca de Studi Religiosi, 4). ISBN : 88-86919-12-3.

L'ouvrage de Jan N. Bremmer, Greek Religion, publié en 1994 dans les suppléments de la revue Greece \& Rome, s'adresse à un public assez large, respectant en cela la politique éditoriale de la revue anglaise. La traduction italienne de l'ouvrage, qui a été publiée en 2002 à l'initiative de Giovanni Casadio, a le mérite indiscutable d'avoir mis à la disposition d'un public encore plus large ce survey que $\mathrm{J}$. Bremmer a conçu comme une mise à jour de l'étude de référence de Walter Burkert (Griechische Religion der archaischen und klassischen Epoche, Stuttgart, 1977). L'identification du destinataire est un élément essentiel afin de cerner les nombreuses qualités et les quelques limites de cette présentation de la religion grecque, où l'A. conjugue un admirable effort de synthèse et l'examen de l'historiographie récente, sans renoncer pour autant à développer ses propres arguments. L'approche éclectique du polythéisme grec que l'A. revendique dès la préface sert parfaitement ce propos.

Comme l'avait déjà fait W. Burkert, J. Bremmer concentre son attention sur la religion grecque aux époques archaïque et classique. Dans le premier chapitre, l'A introduit les traits généraux du polythéisme grec, dont il souligne l'imbrication totale (« embeddedness », traduit en italien par «radicamento ») avec la société dans son ensemble, le caractère public et communautaire, l'absence d'establishment religieux et de textes sacrés. Il prend soin de signaler constamment au lecteur l'interpénétration et la cohérence de toutes les composantes qui, pour les besoins de l'exposition, vont faire l'objet de différents chapitres : les dieux (Chap. II), les sanctuaires (Chap. III), le rituel (Chap. IV), la mythologie (Chap. V). Le parcours initiatique des jeunes filles et les rituels féminins sont traités dans une section autonome (Chap. VI : «Gender », traduit en italien par "Identità sessuale »), à partir des perspectives ouvertes par l'application des notions de gender à l'étude de la religion grecque.

Même si l'A. plaide en faveur d'une plus grande attention à la diachronie, il n'en tient compte que dans la partie conclusive de l'ouvrage. Dans le dernier chapitre, qui porte l'intitulé significatif de "Transformations », J. Bremmer fait dériver les mystères d'Éleusis de la désintégration des rites archaïques de puberté; parmi les changements internes à la religion grecque, une place privilégiée est faite à l'orphisme, dont l'A souligne les liens étroits avec le dionysisme, sans oublier de mentionner les nouveautés apportées par la découverte du papyrus de Derveni. La critique interne au sacrifice sanglant, l'ascension des cultes extatiques et la diffusion des pratiques magiques prépareraient dès la fin de la période classique, à en croire l'A., la crise du polythéisme traditionnel. Dans l'appendice, il retrace rapidement le cheminement de la religion grecque à partir de ses origines indoeuropéennes, redimensionne toute prétendue influence crétoise pendant la phase mycénienne, pour privilégier l'idée - tout aussi répandue et à certains égards également contestable - d'une religion grecque qui dériverait de l'Orient, à l'époque archaïque, la plupart de ses éléments constitutifs.

J. Bremmer s'exprime, à juste titre, en faveur d'une approche plus attentive aux spécificités locales des faits religieux grecs, que ce soit au niveau du panthéon (p. 9 ,

\footnotetext{
${ }^{1}$ Nous suivons la pagination de l'édition italienne.
} 
29) ou bien du rituel (cf., à propos des Thesmophories, p. 124). Il critique le schématisme de l'opposition entre divinités olympiennes et chthoniennes (p. 32, p. 50-51) et juge univoques les positions respectives de W. Burkert et de J.-P. Vernant concernant un problème aussi complexe que celui du sacrifice (p. 75-76). Cependant, un certain schématisme n'est pas absent de l'ouvrage de J. Bremmer. En effet, il dresse le tableau d'un panthéon panhellénique qui serait structuré de façon régulière autour de la dialectique entre l'ordre et le désordre, et où les divinités de l'ordre (Zeus, Athéna et Apollon) seraient situées au cour de la cité et les divinités « excentriques » (Déméter, Dionysos, Poséidon) en dehors. Cette organisation hiérarchique, dont l'A. cherche et trouve une représentation iconographique sur le vase de Sophilos (p. 33, 43-44), lui paraît être confirmée non seulement par la localisation des sanctuaires, mais également par le choix des victimes sacrificielles (cf. p. 72, sur l'« excentricité » des porcins offerts à Déméter et Dionysos) et par le déroulement des rituels (sur la rupture de l'ordre social qui caractériserait les fêtes en l'honneur de Dionysos, cf. p. 40-41). Contrairement à ce qu'il préconise, l'A. se tourne vers une interprétation généralisante du panthéon et ne résiste pas à la tentation d'ériger en norme absolue des cas spécifiques ( $c f$., par exemple, p. 43, p. 72 sur la nature « déviante » des victimes offertes à Aphrodite, à savoir les oiseaux, alors que l'étude de V. Pirenne-Delforge, L'Aphrodite grecque, Athènes/Liège, 1994, p. 381-393, montre bien la typologie variée des sacrifices, sanglants et non sanglants, en l'honneur de la déesse). Concernant la prétendue «excentricité » de Déméter que W. Burkert explique par la fonction agricole, alors que l'A. préfère l'attribuer aux accointances de la déesse avec le monde féminin (p. 39), il s'agit d'une image univoque à laquelle il serait souhaitable de renoncer tout court.

En parcourant de façon claire et concise les grandes lignes de l'historiographie, J. Bremmer introduit son lecteur aux débats autour du «mythe » et du « rite », et met en évidence la complexité de leurs rapports mutuels. Une autre catégorie non indigène, celle d'« initiation », retient particulièrement son attention et se voit attribuer une place centrale dans sa vision de la religion grecque. On aimerait signaler que, dernièrement, les spécialistes se sont davantage interrogés sur la pertinence de cette catégorie ( $c f$. C. CALAME, «Le rite d'initiation tribale comme catégorie anthropologique », RHR 220, 2003, p. 5-62; D.B. DodD, C.A. FaraOne (éds), Initiation in Ancient Greek Rituals and Narratives. New critical perspectives, London/New York, 2003).

Concernant la traduction italienne, il nous revient de signaler la présence de plusieurs coquilles (par exemple, p. 9 n. 1: «Acacia» au lieu d'« Acaia »; p. 39: «Demetria » au lieu de «Demetra »; p. 80 n. 163 : «divinizazione » et p. 184 « divinizzazione », au lieu de «divinazione »), de quelques erreurs (p. 62 : «Esculapio » au lieu d'« Asclepio »), et de certains équivoques (p. 66 : alors que J. Bremmer rappelle que le rituel n'est pas une catégorie indigène en Grèce, l'italien «categoria innata » ne traduit que de façon trompeuse l'anglais «native category »; p. 80 : sur le vase décrit par l'A., Arès assiste non pas «al parto di Afrodite », mais à la naissance, «birth », de la déesse; p. 84 : un sacrifice se terminant par un banquet devient un «sacrificio a conclusione del banchetto »). On aurait aimé trouver, à la fin du volume, une bibliographie des ouvrages cités : en effet, le système des notes de bas de page, où les références ne sont citées in extenso que la première fois, ne permet pas au lecteur de profiter pleinement de cette mise à jour bibliographique qui constitue l'une des richesses de l'ouvrage de J. Bremmer (dans l'édition anglaise, il n'y a pas non plus de bibliographie finale et les notes sont placées à la fin de chaque chapitre). On ne peut que regretter l'absence d'un index, dont la présence reste l'un des atouts majeurs de l'édition anglaise. En revanche, il faut signaler que l'édition italienne tient compte d'une mise à jour bibliographique et qu'elle est enrichie par une note bio-bibliogra- 
phique de l'A., de même que par une post-face de Giovanni Casadio, professeur en Histoire des religions à l'Université de Salerno.

Au nom de la vocation comparatiste de l'histoire des religions, G. Casadio examine à l'aune de ce paramètre les parcours respectifs de A. Brelich, J.-P. Vernant et J. Bremmer; après avoir durement critiqué l'isolationnisme de la culture française, où les études sur la religion grecque se seraient de plus en plus renfermées dans un parcours «idiographique », il souligne les bienfaits de la position éclectique de J. Bremmer qui lui paraît plus ouvert à reconnaître les analogies entre l'expérience du polythéisme grec et celles des religions monothéistes. G. Casadio se déclare intimement persuadé de l'unité fondamentale de la nature humaine et de ses manifestations symboliques. C'est là un article de foi que le recenseur avoue ne pas pouvoir partager. Quoi qu'il en soit, on le remerciera pour avoir contribué à la diffusion de l'ouvrage de J. Bremmer. En effet, cette étude synthétique constitue une introduction valable et actualisée à l'étude de la religion grecque et, dans la mesure où elle opère une efficace mise en perspective méthodologique, sa lecture s'avère des plus utiles également pour un public spécialisé.

Louise BRUIT ZAIDMAN, Les Grecs et leurs dieux. Pratiques et représentations religieuses dans la cité à l'époque classique, Paris, Armand Colin, 2005. 1 vol. $16 \times 24$ cm, 198 p. (Collection U - Histoire). ISBN : 2-200-26230-2.

On se souvient que l'A. a publié récemment une fort belle étude sur la piété des Grecs (Le commerce des dieux, Paris, La Découverte, 2000; c.r. dans Kernos 15, p. 389395). L'ouvrage que voici diffère, à bien des égards, du précédent, mais il s'inscrit manifestement dans une même visée : explorer la relation que les Grecs ont entretenue avec leurs dieux en observant leurs différentes pratiques ainsi que les représentations mentales qui les sous-tendent. Si dieux et déesses sont bien présents à chaque page du livre et qu'on y voit défiler toutes les grandes figures du panthéon, aux côtés de quelques divinités mineures et de héros, il ne faut pas s'attendre à trouver ici une succession de petites monographies vouées à chacune de ces entités divines. Comme il est précisé dans une substantielle introduction (p. v), la démarche consistera à «partir de l'expérience du religieux telle qu'elle imprègne les individus au fil de leur vie », étant entendu que cette expérience est étroitement solidaire du système religieux complexe lié à la cité. Pour réaliser ce dessein, sept thématiques ont été sélectionnées qui forment autant de chapitres, longs pour la plupart d'une bonne vingtaine de pages : "Vie familiale et religion domestique », "Fêtes de village et célébrations civiques », "Interroger les dieux », "Sanctuaires de guérison et dieux guérisseurs », "Après la mort », "Des dieux redoutables: colères et châtiments ", "Cultiver les dieux. Offrandes, sacrifices, prières ». Comme il ressort de ces intitulés limpides, c'est bien à un survol panoramique de la religion grecque que le lecteur est convié. La revue, certes, ne se veut pas complète, mais elle est suggestive et largement représentative de l'expérience des Grecs. Concourent à cette réussite non seulement le choix des thèmes retenus, mais plus encore la façon de les traiter. Celle-ci témoigne, on n'en est pas surpris, d'une compétence très au fait des recherches récentes et elle se signale en outre par une méthode vivante d'exposition qui s'avère particulièrement bien adaptée au but poursuivi. Introduit par des généralités qui cadrent la matière traitée, chaque chapitre, en effet, se développe en une série de sujets particuliers, mis en relief par des sous-titres et systématiquement illustrés par des textes puisés chez les Anciens. 